\title{
Generalized binomial state: Nonclassical features observed through various witnesses and a measure of nonclassicality
}

\author{
Kathakali Mandal ${ }^{\S}$, Nasir Alam ${ }^{\dagger}$, Amit Verma ${ }^{\S}$, Anirban Pathak ${ }^{\dagger}$ and J. Banerji* \\ §Jaypee Institute of Information Technology, Sector-128, Noida, UP-201304, India \\ $\dagger$ Jaypee Institute of Information Technology, A-10, Sector-62, Noida, UP-201309, India \\ *Physical Research Laboratory, Navrangpura, Ahmedabad-380 009, India
}

November 27, 2018

\begin{abstract}
Experimental realization of various quantum states of interest has become possible in the recent past due to the rapid developments in the field of quantum state engineering. Nonclassical properties of such states have led to various exciting applications, specifically in the area of quantum information processing. The present article aims to study lower- and higher-order nonclassical features of such an engineered quantum state (a generalized binomial state based on Abel's formula). Present study has revealed that the state studied here is highly nonclassical. Specifically, higher-order nonclassical properties of this state are reported using a set of witnesses, like higher-order antibunching, higher-order sub-Poissonian photon statistics, higher-order squeezing (both Hong Mandel type and Hillery type). A set of other witnesses for lower- and higher-order nonclassicality (e.g., Vogel's criterion and Agarwal's A parameter) have also been explored. Further, an analytic expression for the Wigner function of the generalized binomial state is reported and the same is used to witness nonclassicality and to quantify the amount of nonclassicality present in the system by computing the nonclassical volume (volume of the negative part of the Wigner function). Optical tomogram of the generalized binomial state is also computed for various conditions as Wigner function cannot be measured directly in an experiment in general, but the same can be obtained from the optical tomogram with the help of Radon transform.
\end{abstract}

\section{Introduction}

With the advent of quantum state engineering [1-4] and quantum computation [5, 6], much attention has been given to the nonclassical properties of quantum states $7-12]$. The reason behind this intense attention is obvious as the nonclassical states being the states having no classical analogue must be essential for performing tasks that are impossible in the classical world (e.g., teleportation, densecoding, unconditionally secure quantum key distribution). In other words, nonclassical states which are characterized by the negative values of Glauber-Sudarshan $P$-function, can only establish quantum supremacy 13,14$]$.

Well known nonclassical properties are squeezing, antibunching and entanglement. These nonclassical features have been reported in various physical systems including optical couplers [15, 16], Bose-Einstein condensates [17], optomechanical systems 1820 , and in many families of quantum states [21 24]. Further, applications of squeezed states are known in the context of LIGO experiment (which has been used successfully to detect gravitational wave) 25, 26], continuous variable quantum key distribution [27 [30]; application of entanglement is known in the context of quantum teleportation and quantum cryptography [31 33], and antibunching is known to be useful in characterizing single photon sources [10,34 used in quantum cryptography [35]. In short, nonclassical features of quantum states are very important and the same has been studied for various families of quantum states. One such family of quantum states is called intermediate states 8 10. These states are interesting because any state of these family can be reduced to various other quantum states at different limits of the parameters which define an intermediate state.

First intermediate state was formally introduced by Stoler et al., in $1985[36$. The state is referred to as Binomial 
state (BS) [36] and can be defined as

$$
|p, M\rangle=\sum_{n=0}^{M} B_{n}^{M}(p)|n\rangle=\sum_{n=0}^{M}\left[{ }^{M} C_{n} p^{n}(1-p)^{M-n}\right]^{1 / 2}|n\rangle,
$$

where $B_{n}^{M}(p)$ is the probability amplitude of the binomial state which corresponds to the occurrence of $n$ photons with equal probability $p$ obtained in $M$ independent ways [36. Mathematically, the binomial state is equivalent to a molecular system having same photon emitting probability $p$ from the different energy levels of the excited states of the molecule which undergoes the $\mathrm{M}$ level vibrational relaxation [37]. Binomial state being an intermediate state, reduces to various existing states at different limits. For example, it reduces to a (a) vacuum state $|0\rangle$ (if $p \rightarrow 0$, $M \rightarrow 0$ ), (b) number state $|n\rangle$ (if $p \rightarrow 1$ and $M$ is finite), (c) coherent state with real amplitude $|\alpha\rangle$ (if $p \rightarrow 0$, $M \rightarrow \infty$ with $p M=\alpha^{2}$ (constant)). It is interesting to note that coherent states are closest to classical states and the number states are the most nonclassical states. Thus, fundamentally different states of electromagnetic field can be obtained as limiting cases of BS. Naturally, properties of BS has been studied since long 38].

The interest on the BS is not restricted to the state of the form Eq. (1), it has been extended to various variants of BS, too. Specifically, in Refs. [39 40] negative binomial state was proposed, and subsequently its properties were studied in Refs. [10]. Similarly, reciprocal binomial state was introduced in Ref. [12] and studied in [9 10]. Further, a couple of generalized binomial states (GBS) $]^{1}$ have been proposed [37] and their nonclassical properties have also been investigated [9]10]. More interestingly, possible applications of GBS have been explored in the field of quantum computation 41. In what follows, we aim to study lower- and higher-order nonclassicality of a particular version of GBS which was introduced by Fan and Liu [37] and referred to as a new GBS (NGBS).

In the introductory work of Fan and Liu, a few nonclassical properties of this state (e.g., squeezing, and subPoissonian photon statistics) was investigated. These were lower-order nonclassical features, but no attention was paid to higher-order nonclassical properties of NGBS. It was natural, as at that time, higher-order nonclssicality was not of much interest (of course Lee [42] and Hong and Mandel (HM) [43, had already introduced the notion of higher-order antibunching and higher-order squeezing [44]), but in the recent past higher-order nonclassicality has been reported theoretically in 8.21 and experimentally in [45 46. Further, their applications (specially applications of higher-order entanglement) have been reported in establishing quantum supremacy [47]. Motivated by these facts (specially the fact that higher-order nonclassical properties of NGBS has not yet been studied), and the fact that a bunch of interesting lower-order nonclassical properties of NGBS is yet to be investigated, in what follows we study the possibilities of observing various lower-order and higher-order nonclassical features for NGBS. Specifically, we report higher-order antibunching (HOA), higher-order sub-Poissonian photon statistics (HOSPS) and higher-order squeezing (HOS) of NGBS. We also report nonclassical features present in NGBS using Agarwal Tara criterion, Vogel's criterion and Wigner function. These criteria of nonclassicality are essentially witnesses of nonclassicality as they do not provide any quantitative estimation of the amount of nonclassicality present in the system. To address this issue, we have also computed nonclassical volume (volume of the negative part of Wigner function) which is a measure of the amount of nonclassicality.

Here it would be apt to note that to obtain the nonclassical volume, we have first derived an analytic expression for the Wigner function of any finite superposition of Fock states (qudits), and have subsequently used that to quantify the nonclassicaity present in NGBS. Further, NGBS may be prepared by generalizing the exisiting proposals for experimental generation of a family of BS including some GBSs (cf. [12, 48 50]) and the schemes used for the experiemntal realization of other intermediate states like photon added coherent state [51]. If NGBS can be prepared, then the prepared state is to be characterized by state tomography. Keeping this possibility in mind, optical tomographs for NGBS are also produced in this work.

The rest of the paper is organized as follows. In the next section, we formally introduce NGBS and describe some identities related to the moments of NGBS. In Section 3, we introduce a set of moment-based criteria of nonclassicality, and establish the existence of nonlcassicality in NGBS by using those criteria. A quantitative measure of nonclassicality is also provided in the form of nonclassical volume of Wigner function. In Section 3.8 , we provide optical tomograms for NGBS. Finally, the paper is concluded in Section 4.

\footnotetext{
${ }^{1}$ If BS can be obtained as a special (limiting) case of a quantum state, then that quantum state would be referred to as GBS.
} 


\section{Generalized binomial state of our interest and analytic expressions of moments}

The NGBS introduced by Fan and Liu [37] is defined as

$$
|M, p, q\rangle=\sum_{n=0}^{M} B_{n}^{M}(p, q)|n\rangle,
$$

where

$$
B_{n}^{M}(p, q)=\left[\frac{p}{1+M q}^{M} C_{n}\left(\frac{p+n q}{1+M q}\right)^{n-1}\left(1-\frac{p+n q}{1+M q}\right)^{M-n}\right]^{1 / 2},
$$

with $n=0,1,2,3, \ldots . M$ and $0<p<1$. Mathematical expression of NGBS was given by Fan and Liu by using the Abel's generalization of the binomial formula, i.e., $p$ is replaced by $\frac{p+n q}{1+M q}$ in Eq. (1), where the value of $q$ may be positive, zero or negative but in order to satisfy the condition for the probability amplitude, $B_{n}^{M}(p, q)>0, q$ must

obey $q \geq \operatorname{Max}\left\{-\frac{p}{M},-\frac{(1-p)}{M}\right\}$. From Eq. 3 it is clear that when $q=0$, NGBS 22 reduces to BS 1 . However, it is interesting to investigate the change of nonclassical properties for other values of $q$. Physically, the objective behind the generalization involved here was that the photon emitting probability $p$ of a molecular system is different for all the energy levels of an excited state during a nonlinear process [37]. This is why the factor $q$ was introduced to model the generalized process. It is also noted that for large values of $M, q$ is small for the fixed values of $p$. Therefore, from the experimental point of view the NGBS appears to be more general and more realistic state.

There exists a large number of nonclassical criteria. Many of them are based on the moments of creation and annihilation operators $\left(a^{\dagger}\right.$ and $a$ ). This section is focused on getting a general expression of moments of annihilation and creation operators for NGBS. Repeatedly applying the annihilation operator on NGBS we obtain

$$
\begin{aligned}
a|M, p, q\rangle & =\left[\frac{p M}{(1+M q)^{M}} \sum_{n=0}^{M} \frac{(M-1) !}{(n-1) ![(M-1)-(n-1) ! !}(p+n q)^{n-1}(1-p+(M-n) q)^{M-n}\right]^{1 / 2}|n-1\rangle, \\
a^{2}|M, p, q\rangle & =\left[\frac{p M(M-1)}{(1+M q)^{M}} \sum_{n=0}^{M} \frac{(M-2) !}{(n-2) ![(M-2)-(n-2)] !}(p+n q)^{n-1}(1-p+(M-n) q)^{M-n}\right]^{1 / 2}|n-2\rangle,
\end{aligned}
$$

and

$$
a^{l}|M, p, q\rangle=\left[\frac{M !}{(M-l) !} \sum_{n=0}^{M}{ }^{M-l} C_{n-l} \frac{p}{1+M q}\left(\frac{p+n q}{1+M q}\right){ }^{n-1}\left(1-\frac{p+n q}{1+M q}\right) M-n\right]^{1 / 2}|n-l\rangle .
$$

Eq. (6) implies

$$
\langle M, p, q| a^{\dagger k}=\left\langle n^{\prime}-k\right|\left[\frac{M !}{(M-k) !} \sum_{n^{\prime}=0}^{M}{ }^{M-k} C_{n^{\prime}-k} \frac{p}{(1+M q)}\left(\frac{p+n^{\prime} q}{1+M q}\right) n^{\prime}-1\left(1-\frac{p+n^{\prime} q}{1+M q}\right){ }^{M-n^{\prime}}\right]^{1 / 2} .
$$

Therefore,

$$
\begin{aligned}
&\left\langle a^{\dagger k} a^{l}\right\rangle=\frac{p M !}{(1+M q)}\left[\sum_{n=0}^{M} \frac{1}{(n-l) !} \sqrt{\frac{1}{(M-n) !(M-n+l-k) !}\left(\frac{p+n q}{1+M q}\right)^{n-1}\left(\frac{p+(n-l+k) q}{1+M q}\right)^{n-l+k-1}}\right. \\
& \times\left.\sqrt{\left(1-\frac{p+n q}{1+M q}\right)^{M-n}\left(1-\frac{p+(n-l+k) q}{1+M q}\right)^{M-n+l-k}}\right]
\end{aligned}
$$

In what follows, we will see that the above analytic expressions will essentially lead to analytic expressions for various witnesses of nonclassicality. 


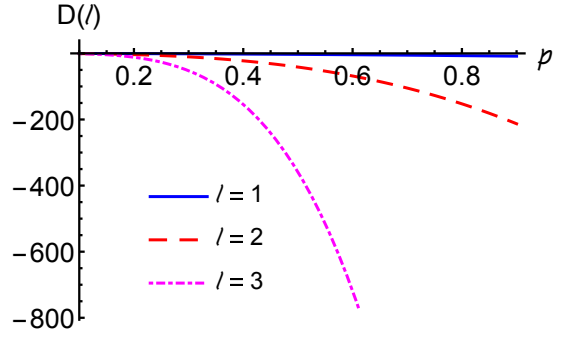

(a)

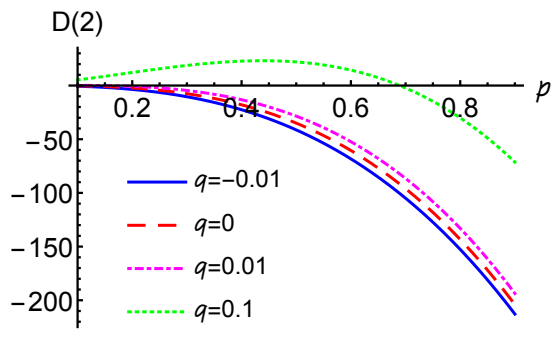

(b)

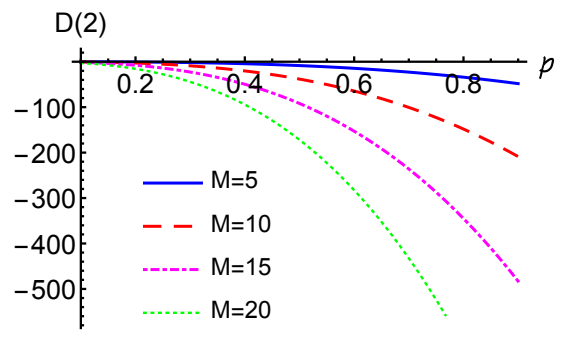

(c)

Figure 1: (Color online) Variation of HOA for NGBS is shown here with probability $p$ for the fixed values of (a) $M=10$ and $q=-0.02$ with different order number, (b) $M=10$, and $l=2$ with different values of $q$, and (c) $l=2$ and $q=-0.005$ with different values of $M$.

\section{Nonclassical properties of NGBS}

\subsection{Higher-order antibunching}

The phenomena of antibunching is closely related to the photon statistics of a state. This phenomenon corresponds to a physical situation involving two photons (or 2 modes), in which the probability of getting two photons simultaneously is less than the probability of getting them separately (one-by-one). Generalization of this idea into multi-photon regime leads to the notion of HOA. In fact, in 1990, Lee 42 introduced the concept of the HOA using the theory of majorization. Lee's criterion for HOA was subsequently modified by Ba An [52] and Pathak and Garcia, [53. As per the criterion of Pathak and Garcia, a quantum state is considered to be higher-order antibunched if it satisfies the following inequality $[53$

$$
D(l)=\left\langle N^{(l+1)}\right\rangle-\langle N\rangle^{l+1}=\left\langle a^{\dagger l+1} a^{l+1}\right\rangle-\left\langle a^{\dagger} a\right\rangle^{l+1}<0,
$$

where $N=a^{\dagger} a$ is the number operator and $N^{(l+1)}=a^{\dagger l+1} a^{l+1}$ is the $l$ th order factorial moment, respectively. Eq. (9) corresponds to the $l$ th order antibunching criterion. For $l=1$, it reduces to the lower-order (conventional) antibunching criterion and for $l \geq 2$ it corresponds to higher-order antibunching criterion. In this article, we have investigated HOA using the criterion (9). We have clearly observed the existence of HOA in NGBS (cf. Figs. 1(a)(c)). The negative part of the curves ensures that NGBS satisfies the inequality (9) and hence NGBS is higher-order antibunched. In Figs. 1(a) and (c), we observe that the depth of the HOA witness increases with the increase of order number $l$ and the dimension $M$, whereas in Fig. 1(b), it decreases with $q$ and for the large values of $q$ it becomes positive, i.e., the signature of HOA is found to be lost for large values of $q$.

\subsection{Higher-order sub-Poissonian photon statistics}

Higher-order nonclassical feature associated with the photon statistics of a quantum state of radiation field is usually studied through the witness of HOSPS. Here it may be noted that HOSPS is the higher-order analogue of the frequently investigated sub-Poissonian photon statistics, and HOSPS is observed for a state if higher-order moment of the photon number for that state is found to be less than the corresponding moment for a Poissonian

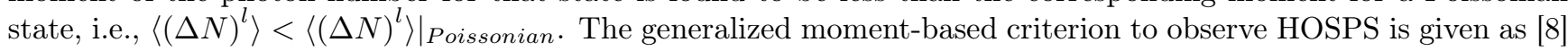

$$
D_{h}(l-1)=\sum_{r=0}^{l} \sum_{k=0}^{r} S_{2}(r, k)^{l} C_{r}(-1)^{r} D(k-1)\langle N\rangle^{l-r}<0
$$

where $S_{2}(r, k)$ is the Stirling number of the second kind. The inequality in Eq. 10 is the condition for the $(l-1)$ th order nonclassicality, and for $l \geq 3$ it leads to the condition for HOSPS. We computed analytic expression for $D_{h}(l-1)$ using Eqs. (8) and (9), and the corresponding results are illustrated in Figs. 2(a)-(c), where the negative parts in figures depict the existence of HOSPS in NGBS. In Figs. 2(a) and (c), we can also observe that the depth of the witness of HOSPS increases with order number $l$ and dimension $M$, but it decreases with parameter $q$ (cf. 


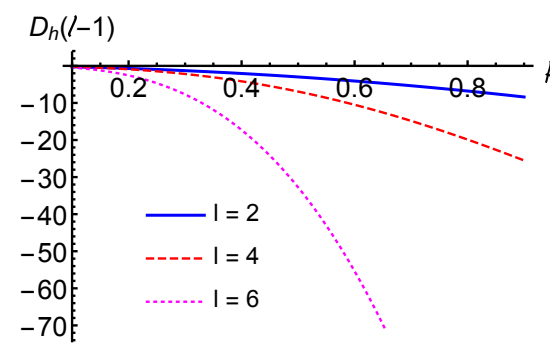

(a)

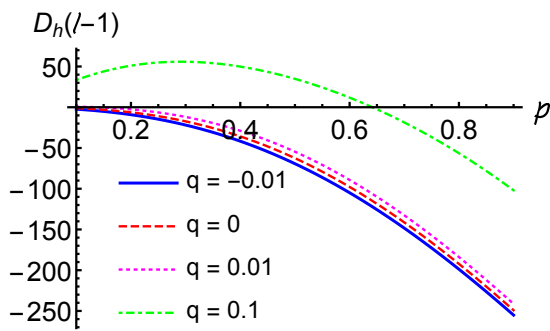

(b)

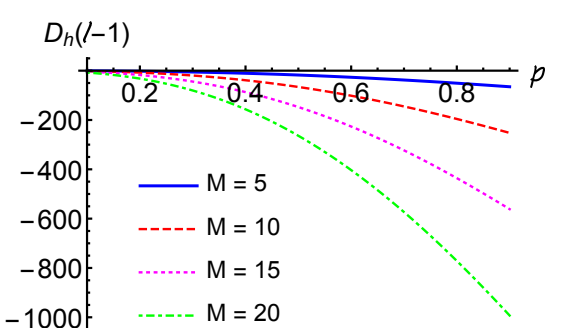

(c)

Figure 2: (Color online) Variation of HOSPS criterion for NGBS is shown here with probability $p$ for the fixed values of (a) $M=10$ and $q=-0.02$ with different order number, (b) $M=10$, and $l=2$ with different values of $q$, and (c) $l=2$ and $q=-0.005$ with different values of $M$.

Fig. 2(b)). From Fig. 2(b), it is observed that for large values of $q$, HOSPS criterion (10) is not satisfied when the probability remains below a certain value. However, it is observed when the probability is greater than that value.

\subsection{Higher-order squeezing}

The squeezing phenomenon originates from the Heisenberg uncertainty relation. The product of fluctuation of two non-commuting operators in Heisenberg uncertainty relation (uncertainty product) has a minimum value. For a coherent state the quadrature variances are equal, and their product possesses minimum value. If the variance of one of the quadrature goes below this value (in order to respect the Heisenberg uncertainty relation the other quadrature must be greater than this value), the corresponding quadrature is considered to be squeezed. The higherorder counterpart of the squeezing is higher-order squeezing. There are types of higher-order squeezing, which are frequently used- Hong-Mandel squeezing [43 and Hillery type squeezing or amplitude powered squeezing [54. To begin with, we investigate the possibility of observing Hong-Mandel squeezing which can be described by the following criterion

$$
S_{H M}(n)=\frac{\left\langle(\Delta X)^{n}\right\rangle-\left(\frac{1}{2}\right)_{\frac{n}{2}}}{\left(\frac{1}{2}\right)_{\frac{n}{2}}}<0
$$

where

$$
\left\langle(\Delta X)^{n}\right\rangle=\sum_{r=0}^{n} \sum_{i=0}^{\frac{r}{2}} \sum_{k=0}^{r-2 i}(-1)^{r} \frac{1}{2^{\frac{n}{2}}}(2 i-1) ! !{ }^{r-2 i} C_{k}{ }^{n} C_{r}{ }^{r} C_{2 i}\left\langle a^{\dagger}+a\right\rangle^{n-r}\left\langle a^{\dagger k} a^{r-2 i-k}\right\rangle<\left(\frac{1}{2}\right)_{\frac{n}{2}}=\frac{1}{2^{\frac{n}{2}}}(n-1) ! !,
$$

and the symbol $(x)_{n}$ is the conventional Pochhammer symbol. The inequality in Eq. (11) is investigated analytically using Eqs. (8) and (11) and the corresponding results are depicted in Figs. 3(a)-(c) where it is observed that depth of HOS(HM) increases with M, decreases with q and region of nonclassicality decreases with increasing order number.

Hillery treated the HOS in a different way. Instead of higher-order moment, he introduced amplitude powered quadratures. The variance of this quadrature is used to investigate HOS. The criterion to obtain Hillery's amplitude powered squeezing is described as 18

$$
A_{i, a}=\left(\Delta Y_{i, a}\right)^{2}-\frac{1}{2}\left|\left\langle\left[Y_{1, a}, Y_{2, a}\right]\right\rangle\right|<0,
$$

where $Y_{1, a}=\frac{a^{l}+a^{\dagger l}}{2}$ and $Y_{2, a}=\frac{-i\left(a^{l}+a^{\dagger l}\right)}{2}$ are the amplitude powered quadrature. In this article, we have calculated HOS with amplitude squared squeezing i.e., for $l=2$, using Hillery's HOS criterion (13) and Eq. (8). The result is exhibited in Fig. 3(d) where the negative values of the variances clearly show the existence of HOS of Hillery type in NGBS. 


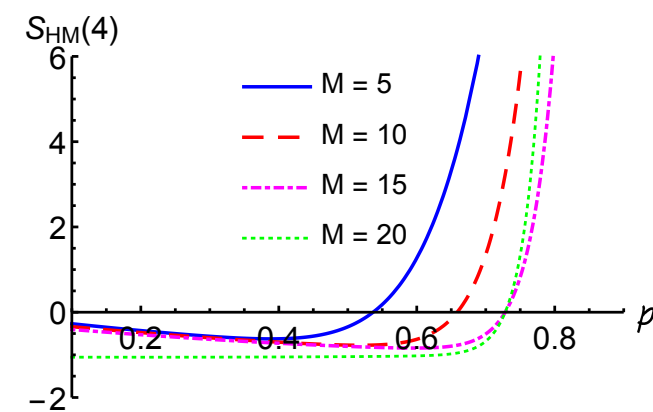

(a)

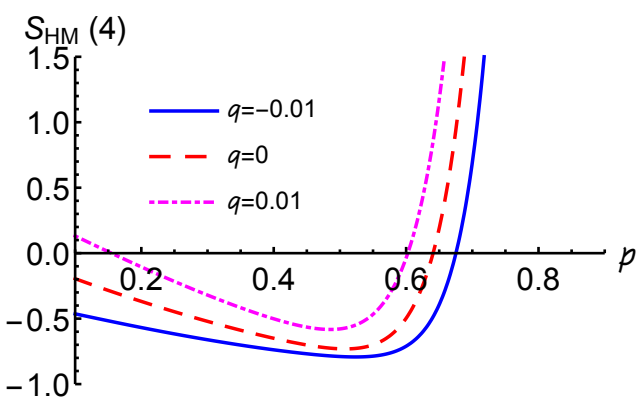

(c)

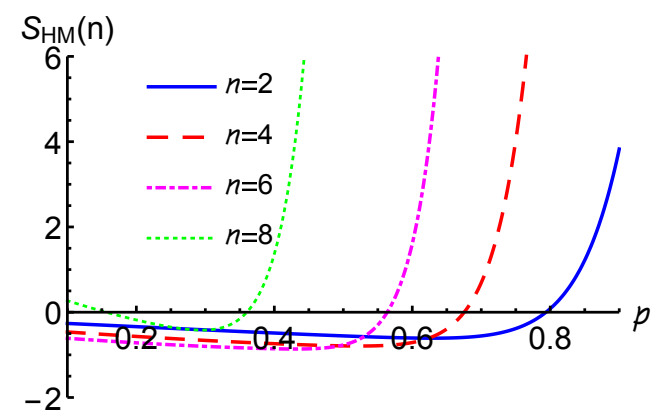

(b)

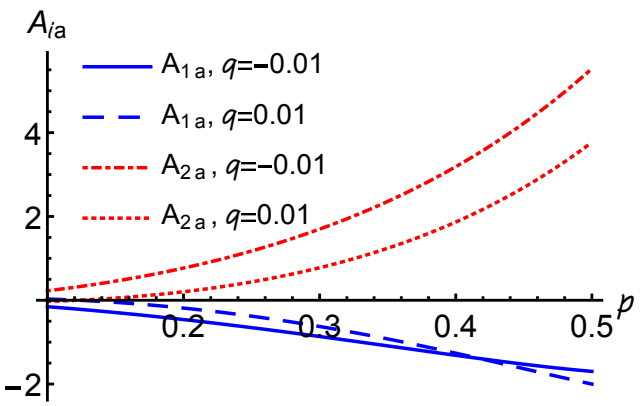

(d)

Figure 3: (Color online) HOS of Hong-Mandel type for NGBS for (a) $q=-0.005$ with different value of $M$, (b) $M=10$ and $q=-0.005$ with different values of $n$ and (c) $M=10$ with different values of $q$. (d) Higher-order squeezing Hillery type for $q=-0.01$ and $M=10$. 


\subsection{Agarwal Tara criterion}

In 1992, Agarwal and Tara 55 introduced a nice criterion to investigate nonclassical phenomenon based on the appropriate generalization of Mandel's Q parameter. This criterion may reveal nonclassicality even when the criteria for the determination of squeezing and sub-Poissonian photon statistics fail to detect any signature of nonclassicality. Specifically, they introduced a criterion for nonclassicality involving the matrix of normally ordered moments $m_{l}=\left\langle a^{\dagger l} a^{l}\right\rangle$ and moments of number distribution $\mu_{l}=\left\langle\left(a^{\dagger} a\right)^{l}\right\rangle$ as follows

$$
A_{n}=\frac{\operatorname{det} m^{(n)}}{\operatorname{det} \mu^{(n)}-\operatorname{det} m^{(n)}}<0
$$

where $\operatorname{det} m^{(n)}$ and $\operatorname{det} \mu^{(n)}$ are the determinant of matrix of normally order moments $m_{n}$ and moments of number distribution $\mu_{n}$, respectively. The matrices $m^{(n)}$ and $\mu^{(n)}$ can be written as

$$
m^{(n)}=\left[\begin{array}{cccc}
1 & m_{1} & \cdots & m_{n-1} \\
m_{1} & m_{2} & \cdots & \vdots \\
\vdots & \vdots & \ddots & \vdots \\
m_{n-1} & \cdots & \cdots & m_{2 n-2}
\end{array}\right] \text { and } \mu^{(n)}=\left[\begin{array}{cccc}
1 & \mu_{1} & \cdots & \mu_{n-1} \\
\mu_{1} & \mu_{2} & \cdots & \vdots \\
\vdots & \vdots & \ddots & \vdots \\
\mu_{n-1} & \cdots & \cdots & \mu_{2 n-2}
\end{array}\right] \text {. }
$$

The nonclassical phenomenon is observed for $-1 \leq A_{n} \leq 0$. Boundary values $A_{n}=0$ and $A_{n}=-1$ correspond to the closest-to-classical (coherent or its mixture) state and Fock (most nonclassical) state, respectively. In brief, for a nonclassical state, $A_{n}$ is always negative and bounded by the value -1 when the state become maximally nonclassical. We have computed analytical expression of $A_{n}$ for particular values of $n$ using Eq. (8) and have investigated the variation of nonclassical phenomenon witnessed through Agarwal-Tara criterion. The results are shown in Figs. 4(a)-(d) where the negative regions of the curves depict nonclassicality. It is observed that negative values of $A_{n}$ increase with probability $p$ and reaches -1 when $p$ approaches unity, indicating that for $p=1$ NGBS is maximally nonclassical. From Figs. 4 (a) and (c), we have seen that for both $A_{2}$ and $A_{3}$ the depth of $A_{n}$ in the negative region decreases with increase of parameter $q$. Similarly, from Figs. 4(b) and (d), we have also observed that the depth of nonclassicality as witnessed through $A_{n}$ increases with $M$. This is consistent with the nonclassical features observed through the other criteria.

\subsection{Vogel's criterion}

In Ref. [56], Vogel et al., derived a criterion for nonclassicality in terms of moments of $a^{\dagger}$ and $a$ operators in the form of $N \times N$ matrix. The determinant of the $N \times N$ matrix is given by

$$
d_{v N}=\left|\begin{array}{ccccc}
1 & \langle a\rangle & \left\langle a^{\dagger}\right\rangle & \left\langle a^{2}\right\rangle & \ldots . . \\
\left\langle a^{\dagger}\right\rangle & \left\langle a^{\dagger} a\right\rangle & \left\langle a^{\dagger 2}\right\rangle & \left\langle a^{\dagger} a^{2}\right\rangle & \ldots . . \\
\langle a\rangle & \left\langle a^{2}\right\rangle & \left\langle a^{\dagger} a\right\rangle & \left\langle a^{3}\right\rangle & \ldots . \\
\left\langle a^{\dagger 2}\right\rangle & \left\langle a^{\dagger 2} a\right\rangle & \left\langle a^{\dagger 3}\right\rangle & \left\langle a^{\dagger 2} a^{2}\right\rangle & \ldots . . \\
\vdots & \vdots & \vdots & \vdots & \ddots
\end{array}\right|_{N \times N} .
$$

For a nonclassical state at least one of the determinants $d_{v N}$ of the matrix 15 , should be negative i.e.,

$$
d_{v N}<0, \text { for } \mathrm{N}=3,4, \ldots .
$$

For $N=2, d_{v N}$ is positive because in this case $d_{v 2}$ represents the incoherent part of the photon number. The negativity of any such sub determinant is the sufficient condition for nonlclassicality. It is also reported that all such moments of $a^{\dagger}$ and $a$ can be realized using beam spliters and homodyne correlation measurements. Here we have reported the result for $N=3$ and 4 in Figs. 5(a)-(d). The plots clearly illustrate the fact that NGBS is highly nonclassical. Further, in consistent with other criteria discussed here, it also shows that with the increase of parameter $q$, the depth and region of nonclassicality witness decrease (cf. Figs. 5(a) and (c)). But this type of consistency is not observed with the increase of dimension $M$. In this case the depth of the nonclassicality witness increases with the increase of dimension $M$ only when the probability is high (cf. Figs. 5(b) and (d)). This is a very general criterion and many of the above mentioned criteria (including Agarwal-Tara criterion and Hillery's criterion of HOS) can be obtained as special cases of this criterion. 


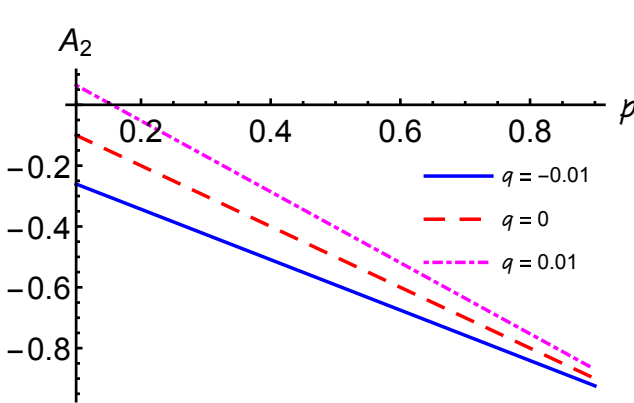

(a)

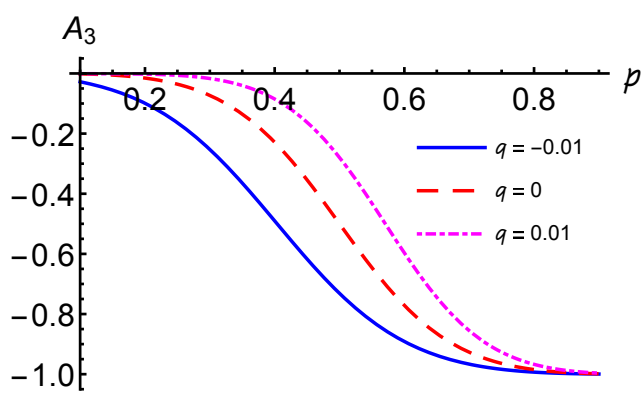

(c)

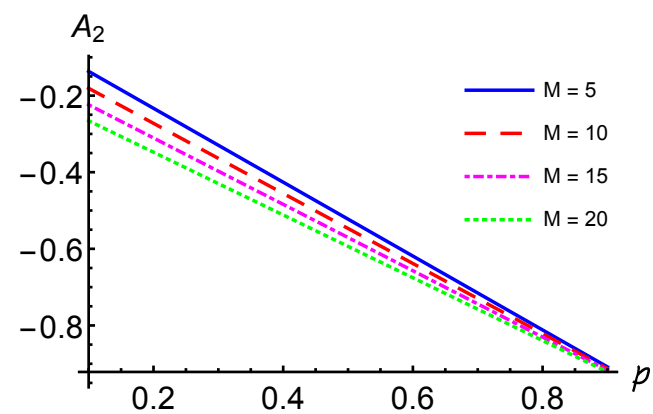

(b)

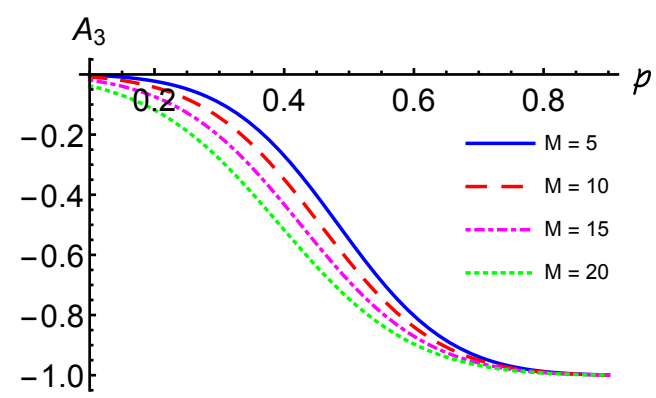

(d)

Figure 4: (Color online) Variation of Agarwal Tara nonclassicality criterion with respect to probability $p$. Variation of $A_{2}$ for (a) $M=10$ and different values of $q$, (b) for $q=-0.005$ and different values of $M$. Similarly, Variation of $A_{3}$ for (c) $M=10$ and different values of $q$, (d) for $q=-0.005$ and different values of $M$.

\subsection{Wigner function}

In this section, we shall investigate Wigner qasiprobability distribution function for NGBS. The NGBS can be written as a finite dimensional Fock superposition state (FSS) and can be viewed as a qudit. Any such Fock superposition state has a general form

$$
|\psi\rangle=\sum_{n=0}^{N} c_{n}|n\rangle,
$$

Such a state has to be nonclassical as we can imagine that this finite dimensional FSS has infinitely many holes in its photon number distribution as for this state $P(i: i>N)=0$, where $P(i)=\left|c_{i}\right|^{2}$ is the probability of finding $i$ th quantum state $i\rangle$ which has $i$ photor ${ }^{2}$ state having holes in their PND is nonclassical in nature. For example, consider an arbitrary state $\rho=\int P(\alpha)|\alpha\rangle\langle\alpha| d^{2} \alpha$ having photon number distribution $P_{n}=\int P(\alpha)|\langle n \mid \alpha\rangle|^{2} d^{2} \alpha$. Since the values $\left.\langle n \mid \alpha\rangle\right|^{2}>0$, therefore, $P_{n} \neq 0$ when $P(\alpha)$ is a true probability density.

NGBS is just a special form of this state (17) where $c_{n}$ should be replaced by Eq. (3). In what follows we provide analytic expressions for Wigner function for finite dimensional FSS of the form [17], in general, and subsequently to witness and quantify nonclassicality present in NGBS by plotting the analytic expression.

In fact, Wigner function and other quasi-probability distributions have been studied for a long time. Naturally, efforts had been made to construct analytic expressions of Wigner function of the states of the form (17). To be precise, in 57 the authors commented, "The Wigner function is usually expressed in an integral form which is not always easy to compute." Keeping that in mind they provided (cf. Eq. (16) of [57]) the following analytic expression of Wigner function:

$$
W(\alpha)=\frac{2}{\pi} \sum_{k=0}^{\infty}(-1)^{k}\langle\alpha, k|\rho| \alpha, k\rangle
$$

\footnotetext{
${ }^{2}$ Any pure quantum state can be expressed as superposition of Fock states. If in photon number distribution (PND) we find $P_{n}=\left|c_{n}\right|^{2}=0$ for any $n$, we say that a hole is created at that position in the photon number distribution.
} 


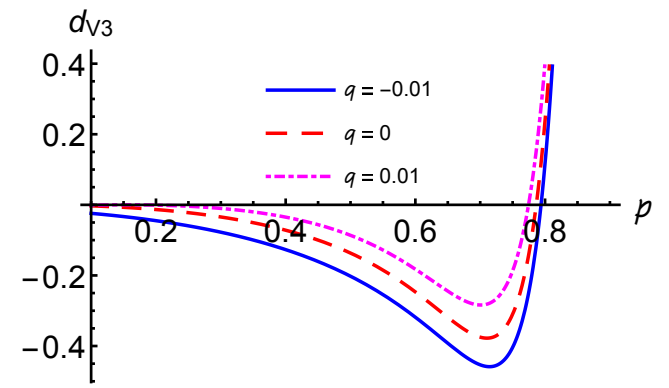

(a)

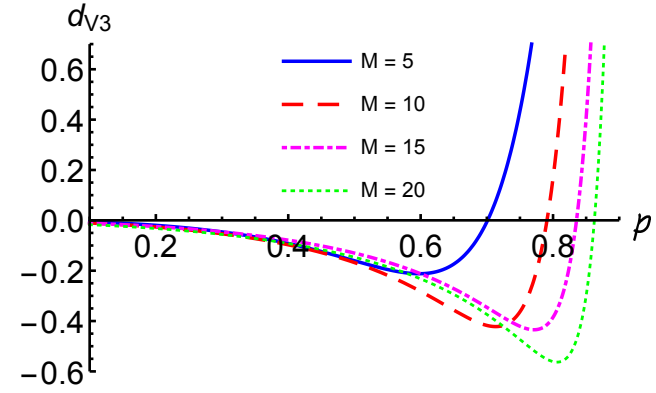

(b)

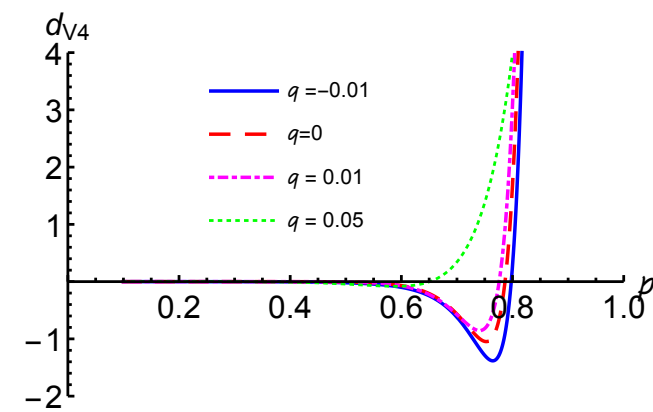

(c)

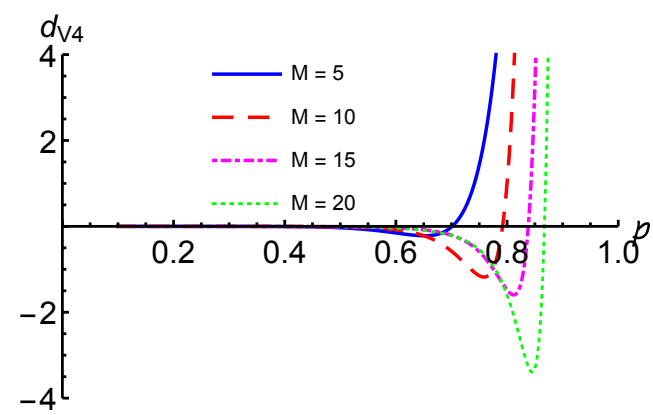

(d)

Figure 5: (Color online) Variation of $d_{v 3}$ with respect to probability $p$ for (a) $M=10$ and different values of $q$, (b) $q=-0.005$ and different values of $M$. Similarly, variation of $d_{v 4}$ with respect to probability $p$ for (c) $M=10$ and different values of $q$ and (d) $q=-0.005$ and different values of $M$. 
where $\rho$ is the density matrix of the state and $|\alpha, k\rangle$ are the displaced number states. Since $|\psi\rangle$ in $(17)$ is a pure state so we have $\rho=|\psi\rangle\langle\psi|$ and consequently,

$$
\begin{aligned}
W(\alpha) & =\frac{2}{\pi} \sum_{k=0}^{\infty}(-1)^{k}|\langle\alpha, k \mid \psi\rangle|^{2} \\
& =\frac{2}{\pi} \sum_{k=0}^{\infty}(-1)^{k}\left|\sum_{n} c_{n}\langle\alpha, k \mid n\rangle\right|^{2} \\
& =\frac{2}{\pi} \sum_{k=0}^{\infty}(-1)^{k}\left|\sum_{n} c_{n}\langle k|D(\alpha)| n\rangle\right|^{2} \\
& =\frac{2}{\pi} \sum_{k=0}^{\infty}(-1)^{k}\left|\sum_{n} c_{n} \chi_{k n}(\alpha)\right|^{2},
\end{aligned}
$$

where $D(\alpha)$ is the displacement operator and $\chi_{k n}(\alpha)=\langle k|D(\alpha)| n\rangle$ which can be expressed in compact form as (cf. Eq. 4.6 of [11])

$$
\chi_{n k}(\alpha)=\left\{\begin{array}{c}
\sqrt{\frac{k !}{n !}} \exp \left(-\frac{|\alpha|^{2}}{2}\right) \alpha^{n-k} L_{k}^{n-k}\left(|\alpha|^{2}\right) \text { if } n \geq k \\
\sqrt{\frac{n !}{k !}} \exp \left(-\frac{|\alpha|^{2}}{2}\right)\left(\alpha^{*}\right)^{k-n} L_{n}^{k-n}\left(|\alpha|^{2}\right) \text { if } n \leq k .
\end{array}\right.
$$

Till now, the above mentioned series expansion of Wigner function was used to investigate the quasi-distribution of FSSs. Here, we would like to note that in contrary to the comment of Cessa and Night in [57, it is often difficult to handle the infinite sum present in the series form of Wigner function. Keeping that in mind, we have derived a compact form of Wigner function of FSS below in integral form and have shown that it is free from the trouble of handling infinity. A detail derivation of the Wigner function is provided in Appendix A. Here we just note the final expression of Wigner function for FSS of above form can be obtained as A-7]

$$
W\left(x, p^{\prime}\right)=\frac{1}{\pi^{\frac{1}{2}}} \sum_{n, n^{\prime}=0}^{N} c_{n}^{*} c_{n^{\prime}} b_{n}^{*} b_{n^{\prime}} \mathrm{e}^{-\left(x^{2}+p^{\prime 2}\right)}(-1)^{n^{\prime}} 2^{n^{\prime}} n !\left(i p^{\prime}-x\right)^{n^{\prime}-n} L_{n}^{n^{\prime}-n}\left(-2 y\left(i p^{\prime}-x\right)\right), \quad n \leq n^{\prime} .
$$

Here we have used $p^{\prime}$ instead of $p$ because the symbol $p$ is already used for probability. Now we can use the formalism presented here to obtain Wigner function for any FSS. However, this paper is focused on NGBS and we restrict ourselves to the construction of Wigner function of NGBS. Specifically, Wigner function of NGBS are computed for various choices of the state parameters. The obtained Wigner functions for the NGBS are shown in Figs. 6(a)-(d). Clearly, there exist negative regions of Wigner function in all the figures and that establishes that the NGBS studied here are nonclassical. However, neither negativity of the Wigner function nor the nonclassicality witnesses studied till now provide any quantitative measure of the nonclassicality. The same may be obtained using nonclassical volume which is described in the following subsection.

\subsection{Nonclassical volume}

From the negativity of the Wigner function, we have obtained the signature of nonclassicality in NGBS. However, the amount of nonclassicality is not yet investigated. There exist several quantitative measures of nonclassicality. Here we may use the most convenient quantitative measure of nonclassicality which is known as nonclassical volume. The nonclassical volume as a measure of quantumness was first introduced by Kenfack and Zyczkowski [58] in 2004. In this particular measure, the volume of the negative part of the Wigner function is considered as the measure of nonclassicality. To be precise, the negative volume associated with a quantum state $|\psi\rangle$ is

$$
\delta(\psi)=\iint\left|W_{\psi}\left(p^{\prime}, q\right)\right| d q d p^{\prime}-1,
$$

where $W_{\psi}\left(p^{\prime}, q\right)$ is the Wigner function of a quantum state $|\psi\rangle$. A non-zero values of $\delta(\psi)$ indicates nonclassical state. As we have a compact expression for the Wigner function, we can use the same to obtain $\delta(\psi)$ for various choices of parameters and investigate how nonclassical volume (or the amount of quantumness) varies with the change of a particular parameter. For example, in Table 1. we have shown the variation of $\delta(\psi)$ with probability $p$ for the fixed values of $M$ in NGBS and it is found that the amount of nonclassicality increases with $p$, which was also indicated by the nonclassicality witnesses, studied in this paper. 


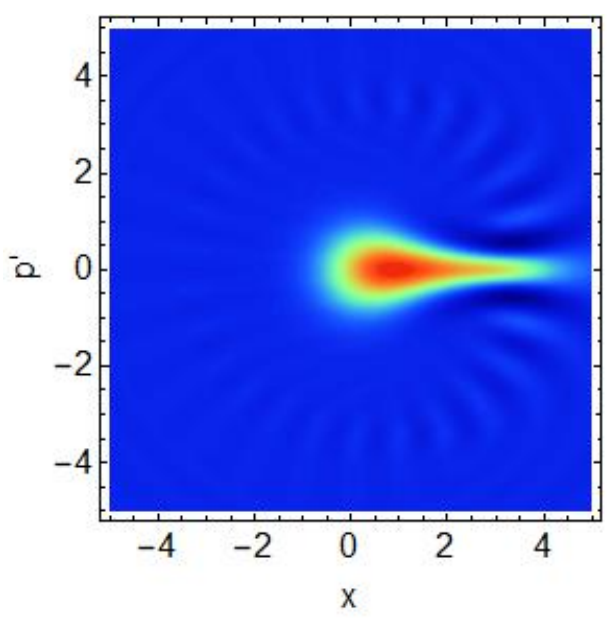

(a)

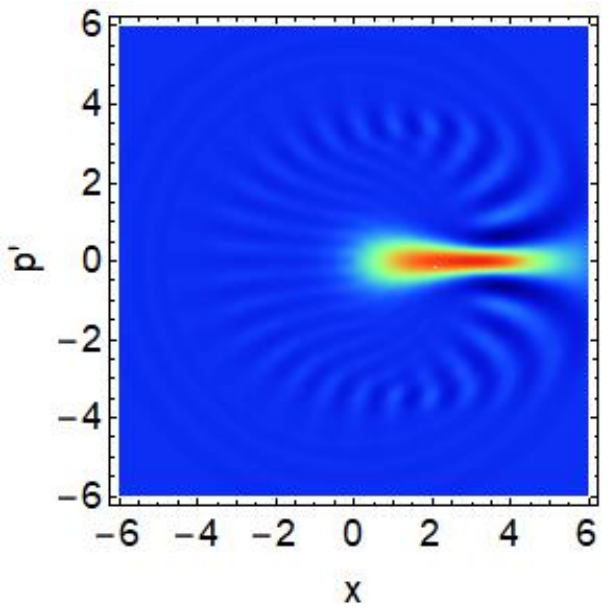

(c)

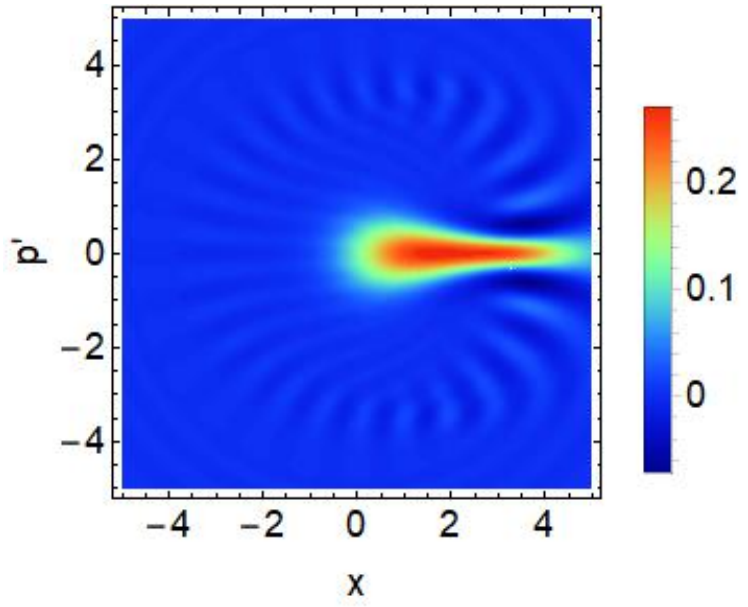

(b)

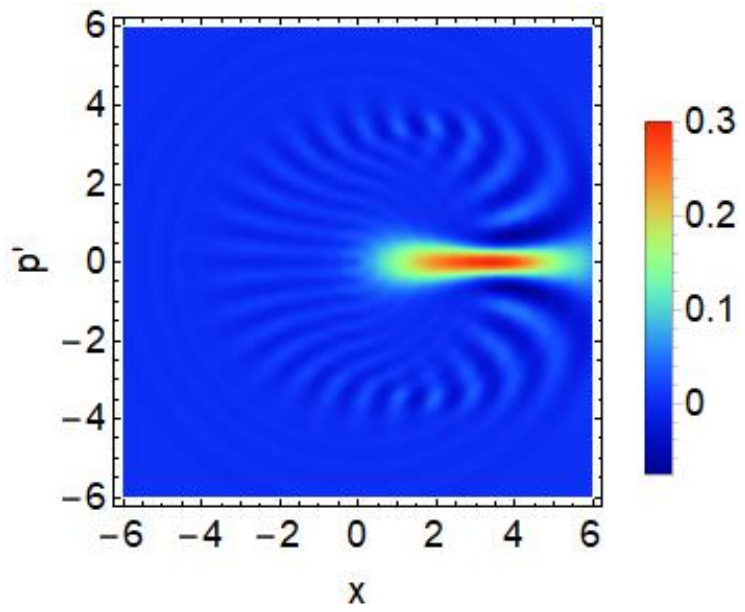

(d)

Figure 6: (Color online) Wigner function for the NGBS for (a) $p=0.2$, (b) $p=0.4$, (c) $p=0.6$ and (d) $p=0.8$, respectively. In all figures $M=25$ and $q=0.5$.

\begin{tabular}{ccc}
\hline Serial Number & Probability $(p)$ & Nonclassical volume $(\delta(\psi))$ \\
\hline \hline 1. & 0.2 & 0.166724 \\
2. & 0.4 & 0.244092 \\
3. & 0.6 & 0.324178 \\
4. & 0.8 & 0.416412 \\
\hline
\end{tabular}

Table 1: Nonclassical volume for NGBS with $q=0.5$ and $M=25$. 


\subsection{Optical tomogram}

There exist some proposals for the direct measurement of Wigner function [59 61]. However, in general, due to its probabilistic nature, the direct measurement of Wigner function is not possible, so we may experimentally measure only by processing of data. Interestingly, tomogram gives the probabilistic description of the quantum state which is accessible for direct measurement. Therefore, Wigner function can be measured by using optical homodyne tomomgraphy 62 . For any quantum state $|\psi\rangle$, the optical tomogram $w_{|\psi\rangle}(X, \theta)$ is the marginal distribution of the field quadrature component $X$ with a rotation by an angle $\theta$ in the quadrature phase space [63]. Mathematically, optical tomogram can be obtained from the Radon transform of the Wigner function. Of late, Filippov and Man'ko 9,63 have reported the following closed form analytic expression for the optical tomogram of finite dimensional FSS (17):

$$
w_{|\psi\rangle}(X, \theta)=\frac{e^{-X^{2}}}{\sqrt{\pi}}\left[\sum_{n=0}^{N} \frac{\left|c_{n}\right|^{2}}{2^{n} n !} H_{n}^{2}(X)+\sum_{n<k} \frac{\left|c_{n}\right|\left|c_{k}\right| \cos \left((n-k) \theta-\left(\phi_{n}-\phi_{k}\right)\right)}{\sqrt{2^{n+k-2} n ! k !}} H_{n}(X) H_{k}(X)\right]
$$

where $c_{j}=\left|c_{j}\right| e^{i \phi_{j}}$ and $H_{j}$ is the Hermite polynomial of degree $j$. The optical tomogram of NGBS has been calculated by using Eqs. (3) and (20). The results are depicted in Figs. 7(a)-(d). The Wigner function for the NGBS can be experimentally verified by optical homodyne tomography technique where the expected tomogram can be obtained theoretically from Eq. 20 .

\section{Conclusion}

Finite dimensional quantum states or qudits have drawn attention of the scientific community as these states have been found to be useful in performing various tasks related to quantum information processing. Construction of such states have become feasible with the rapid development of quantum state engineering. One such interesting finite dimensional quantum state is NGBS which reduces to various states of interest in different limits. Nonclassical properties of this state are studied here with a focus on higher-order nonclassical properties. The analytically obtained expressions for various witnesses of nonclassicality are plotted to establish that NGBS is highly nonclassical and thus can be used for various tasks where nonclassical states are essential. Specifically, the presence of higherorder nonclassical properties (HOA, HOS and HOSPS) in NGBS are shown here for the first time.

Physically, among various intermediate states and the states from the family of binomial and generalized binomial states, NGBS is of particular interest because it is useful when the photon emitting probabilities of a system are not equal hence it is applicable in a more generalized procedure. Physical objective of this generalization procedure is to model a complex physical system such as a molecular system which have different photon emitting probabilities from different energy levels of an excited molecule during any nonlinear procedure. Therefore, this scenario appears to be obvious during any nonlinear process and its experimental realization [37. Thus, the focus of this paper (investigation on NGBS) seems justified from this practical scenario. To investigate nonclassical witnesses and measures in this generalized engineered state, we have used a set of moment-based criteria and also Wigner quasiprobability distribution. Specifically, the moment-based criteria used here to analyze nonclassical features include the criteria for lower- and higher-order antibunching, HOSPS, Hong Mandel HOS, Hillery HOS, Agarwal-Tara criterion and Vogel criterion. The moment-based criteria and quasi-probability distribution, investigated here, have revealed a variety of nonclassical properties of NGBS. In most of the cases, depth of nonclassicality witness is found to increase with dimension but it decreases with the parameter $q$ which is introduced for different photon emitting probabilities in NGBS. However, in a few occasions, namely in the context of HOS Hillery type and Vogel criterion, the results are not always consistent with other criteria. In the case of Vogel's criterion, it is observed that the depth of the nonclassical witness increases with dimension $M$ only for the higher values of probability and this result is not consistent for the lower value of probability (cf. Figs. 5(b) and (c)). On the other hand, in the case of HOS the depth of the inequality increases in the negative region with increase of the parameter $q$ for higher values of probability but it decreases with $q$ for lower values of probability. Hence for lower values of probability $p$, the result is consistent with other criteria (cf. Fig] 3 (d)). The Wigner quasi-probability distribution of the NGBS are reported and the amount of nonclassicality is computed by computing nonclassical volume, which is nothing but the volume of the negative part of the Wigner function. The nonclassical volume (thus the amount of nonclassicality) of NGBS is found to increase with probability $p$. In short, it is established that for higher value of the probability, the amount of nonclassicality in NGBS is high. The nature of optical tomograms for NGBS are explored and reported 


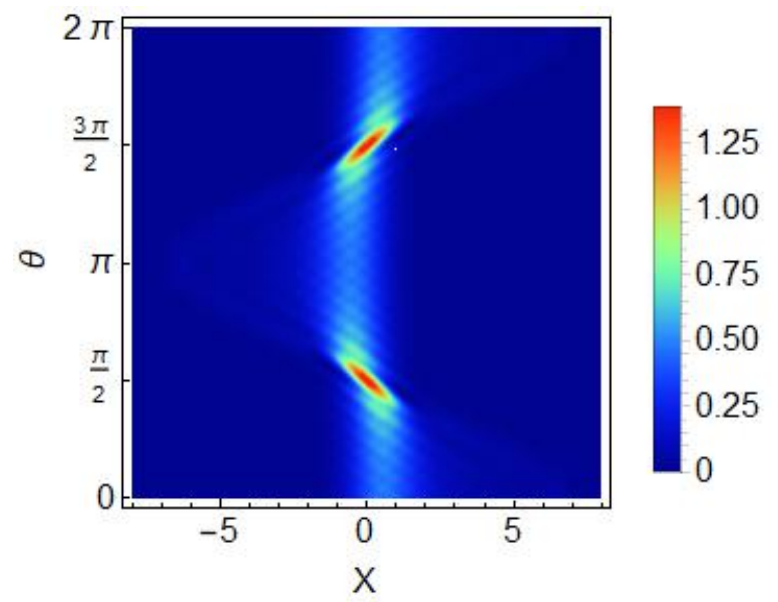

(a)

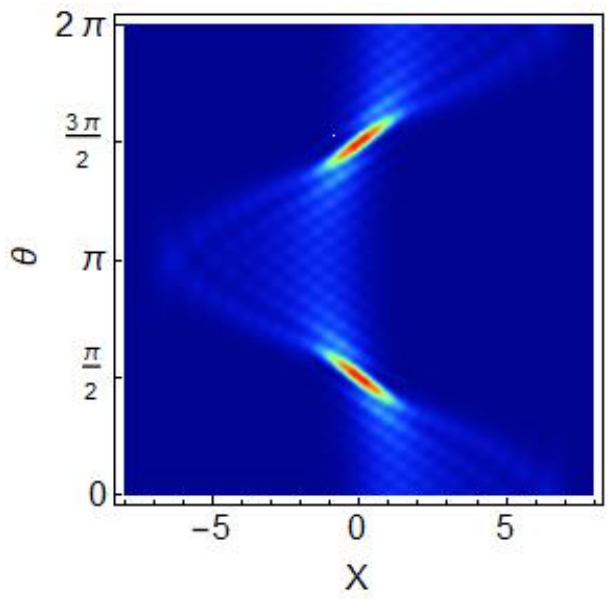

(c)

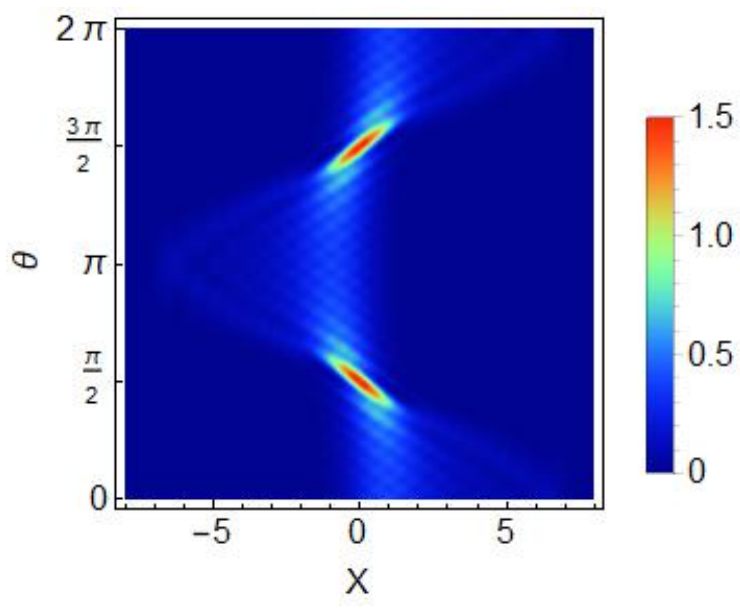

(b)

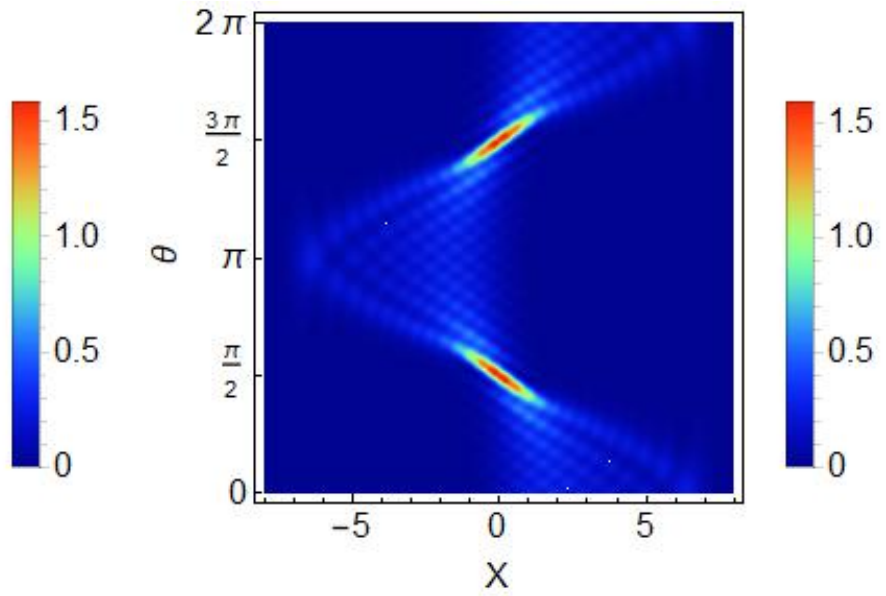

(d)

Figure 7: (Color online) Tomograms for the NGBS for (a) $p=0.2$, (b) $p=0.4$, (c) $p=0.6$ and (d) $p=0.8$, respectively. In all figures $M=25$ and $q=0.5$. 
for different probability. Tomograms can be obtained experimentally and the same may be used to obtain Wigner function with the help of Radon transform. Keeping all these facts in mind, we conclude the paper with the hope that the present observation will be useful in quantum optics and quantum information processing, specifically, in the experimental scenario when the photon emitting probability is not equal.

\section{Acknowledgment:}

A.P. and N.A. thank the Department of Science and Technology (DST), India, for support provided through the DST project No. EMR/2015/000393.

\section{References}

[1] Marcelo A Marchiolli and Wagner Duarte José. Engineering superpositions of displaced number states of a trapped ion. Physica A: Statistical Mechanics and its Applications, 337(1-2):89-108, 2004.

[2] Adam Miranowicz and Wiesław Leoński. Dissipation in systems of linear and nonlinear quantum scissors. Journal of Optics B: Quantum and Semiclassical Optics, 6(3):S43, 2004.

[3] K Vogel, VM Akulin, and WP Schleich. Quantum state engineering of the radiation field. Physical Review Letters, 71(12):1816, 1993.

[4] J Sperling, W Vogel, and GS Agarwal. Quantum state engineering by click counting. Physical Review A, 89(4):043829, 2014.

[5] Michael A Nielsen and Isaac Chuang. Quantum computation and quantum information, 2002.

[6] Anirban Pathak. Elements of quantum computation and quantum communication. CRC Press, 2013.

[7] Stephen M Barnett, Gergely Ferenczi, Claire R Gilson, and Fiona C Speirits. Statistics of photon-subtracted and photon-added states. Physical Review A, 98(1):013809, 2018.

[8] Amit Verma and Anirban Pathak. Generalized structure of higher order nonclassicality. Physics Letters A, 374(8):1009-1020, 2010.

[9] Anirban Pathak and J Banerji. Wigner distribution, nonclassicality and decoherence of generalized and reciprocal binomial states. Physics Letters A, 378(3):117-123, 2014.

[10] Amit Verma, Navneet K Sharma, and Anirban Pathak. Higher order antibunching in intermediate states. Physics Letters A, 372(34):5542-5551, 2008.

[11] Hong-Chen Fu and Ryu Sasaki. Hypergeometric states and their nonclassical properties. Journal of Mathematical Physics, 38(5):2154-2166, 1997.

[12] MHY Moussa and B Baseia. Generation of the reciprocal-binomial state. Physics Letters A, 238(4-5):223-226, 1998.

[13] Aram W Harrow and Ashley Montanaro. Quantum computational supremacy. Nature, 549(7671):203, 2017.

[14] C Neill, P Roushan, K Kechedzhi, S Boixo, SV Isakov, V Smelyanskiy, A Megrant, B Chiaro, A Dunsworth, K Arya, et al. A blueprint for demonstrating quantum supremacy with superconducting qubits. Science, 360(6385):195-199, 2018.

[15] Kishore Thapliyal, Anirban Pathak, Biswajit Sen, and Jan Peřina. Higher-order nonclassicalities in a codirectional nonlinear optical coupler: Quantum entanglement, squeezing, and antibunching. Physical Review A, 90(1):013808, 2014.

[16] Kishore Thapliyal, Anirban Pathak, Biswajit Sen, and Jan Perina. Nonclassical properties of a contradirectional nonlinear optical coupler. Physics Letters A, 378(46):3431-3440, 2014. 
[17] Sandip Kumar Giri, Biswajit Sen, CH Raymond Ooi, and Anirban Pathak. Single-mode and intermodal higher-order nonclassicalities in two-mode bose-einstein condensates. Physical Review A, 89(3):033628, 2014.

[18] Nasir Alam, Kishore Thapliyal, Anirban Pathak, Biswajit Sen, Amit Verma, and Swapan Mandal. Lower-and higher-order nonclassicality in a bose-condensed optomechanical-like system and a fabry-perot cavity with one movable mirror: squeezing, antibunching and entanglement. arXiv preprint arXiv:1708.03967, 2017.

[19] Nasir Alam, Swapan Mandal, and Patrik Öhberg. Approximate analytical solutions of a pair of coupled anharmonic oscillators. Journal of Physics B: Atomic, Molecular and Optical Physics, 48(4):045503, 2015.

[20] Nasir Alam and Swapan Mandal. Nonclassical properties of coherent light in a pair of coupled anharmonic oscillators. Optics Communications, 359:221-233, 2016.

[21] Nasir Alam, Amit Verma, and Anirban Pathak. Higher-order nonclassicalities of finite dimensional coherent states: A comparative study. Physics Letters A, 382(28):1842-1851, 2018.

[22] Nilakantha Meher and S. Sivakumar. Number state filtered coherent states. Quantum Information Processing, 17(9):233, Jul 2018.

[23] Priya Malpani, Nasir Alam, Kishore Thapliyal, Anirban Pathak, V Narayanan, and Subhashish Banerjee. Lower-and higher-order nonclassical properties of photon added and subtracted displaced fock states. arXiv preprint arXiv:1808.01458, 2018.

[24] Nasir Alam, Kathakali Mandal, and Anirban Pathak. Higher-order nonclassical properties of a shifted symmetric cat state and a one-dimensional continuous superposition of coherent states. International Journal of Theoretical Physics, 57(11):3443-3456, Nov 2018.

[25] BP Abbott. Bp abbott, r. abbott, td abbott, mr abernathy, f. acernese, k. ackley, c. adams, t. adams, p. addesso, rx adhikari et al., phys. rev. lett. 116, 061102 (2016). Phys. Rev. Lett., 116:061102, 2016.

[26] BP Abbott, R Abbott, TD Abbott, MR Abernathy, F Acernese, K Ackley, C Adams, T Adams, P Addesso, RX Adhikari, et al. Gw151226: Observation of gravitational waves from a 22-solar-mass binary black hole coalescence. Physical Review Letters, 116(24):241103, 2016.

[27] Daniel Gottesman and John Preskill. Secure quantum key distribution using squeezed states. In Quantum Information with Continuous Variables, pages 317-356. Springer, 2003.

[28] Nicolas J Cerf, Marc Levy, and Gilles Van Assche. Quantum distribution of gaussian keys using squeezed states. Physical Review A, 63(5):052311, 2001.

[29] Lars S Madsen, Vladyslav C Usenko, Mikael Lassen, Radim Filip, and Ulrik L Andersen. Continuous variable quantum key distribution with modulated entangled states. Nature communications, 3:1083, 2012.

[30] Christian Weedbrook, Stefano Pirandola, Raúl García-Patrón, Nicolas J Cerf, Timothy C Ralph, Jeffrey H Shapiro, and Seth Lloyd. Gaussian quantum information. Reviews of Modern Physics, 84(2):621, 2012.

[31] Charles H Bennett, Gilles Brassard, Claude Crépeau, Richard Jozsa, Asher Peres, and William K Wootters. Teleporting an unknown quantum state via dual classical and einstein-podolsky-rosen channels. Physical Review Letters, 70(13):1895, 1993.

[32] Artur K Ekert. Quantum cryptography based on bell's theorem. Physical Review Letters, 67(6):661, 1991.

[33] Charles H Bennett, Gilles Brassard, and Artur K Ekert. Quantum cryptography. Scientific American, 267(4):50-57, 1992.

[34] Anirban Pathak and Amit Verma. Recent developments in the study of higher order nonclassical states. Indian Journal of Physics, 84(8):1005-1019, 2010.

[35] Chitra Shukla, Nasir Alam, and Anirban Pathak. Protocols of quantum key agreement solely using bell states and bell measurement. Quantum Information Processing, 13(11):2391-2405, 2014. 
[36] D Stoler, BEA Saleh, and MC Teich. Binomial states of the quantized radiation field. Optica Acta: International Journal of Optics, 32(3):345-355, 1985.

[37] Hong-Yi Fan et al. New generalized binomial states of the quantized radiation field. Physics Letters A, 264(2-3):154-161, 1999.

[38] Girish S. Agarwal. Quantum Optics. Cambridge University Press, 2012.

[39] GS Agarwal. Negative binomial states of the field-operator representation and production by state reduction in optical processes. Physical Review A, 45(3):1787, 1992.

[40] Stephen M Barnett. Negative binomial states of the quantized radiation field. Journal of Modern Optics, 45(10):2201-2205, 1998.

[41] Rosario Lo Franco, Giuseppe Compagno, Antonino Messina, and Anna Napoli. Quantum computation with generalized binomial states in cavity quantum electrodynamics. International Journal of Quantum Information, 7 (supp01):155-162, 2009.

[42] Ching Tsung Lee. Higher-order criteria for nonclassical effects in photon statistics. Physical Review A, 41(3):1721, 1990.

[43] CK Hong and Li Mandel. Generation of higher-order squeezing of quantum electromagnetic fields. Physical Review A, 32(2):974, 1985.

[44] Sandip Kumar Giri, Kishore Thapliyal, Biswajit Sen, and Anirban Pathak. Nonclassicality in an atom-molecule bose-einstein condensate: Higher-order squeezing, antibunching and entanglement. Physica A: Statistical Mechanics and its Applications, 466:140-152, 2017.

[45] Alessia Allevi, Stefano Olivares, and Maria Bondani. High-order photon-number correlations: a resource for characterization and applications of quantum states. International Journal of Quantum Information, 10(08):1241003, 2012.

[46] Alessia Allevi, Stefano Olivares, and Maria Bondani. Measuring high-order photon-number correlations in experiments with multimode pulsed quantum states. Physical Review A, 85(6):063835, 2012.

[47] Juan Miguel Arrazola, Patrick Rebentrost, and Christian Weedbrook. Quantum supremacy and highdimensional integration. arXiv preprint arXiv:1712.07288, 2017.

[48] C Valverde, AT Avelar, B Baseia, and JMC Malbouisson. Generation of the reciprocal-binomial state for optical fields. Physics Letters A, 315(3-4):213-218, 2003.

[49] R Lo Franco, Giuseppe Compagno, A Messina, and A Napoli. Single-shot generation and detection of a two-photon generalized binomial state in a cavity. Physical Review A, 74(4):045803, 2006.

[50] Rosario Lo Franco, Giuseppe Compagno, Antonino Messina, and Anna Napoli. Efficient generation of n-photon binomial states and their use in quantum gates in cavity qed. Physics Letters A, 374(22):2235-2242, 2010.

[51] Alessandro Zavatta, Silvia Viciani, and Marco Bellini. Quantum-to-classical transition with single-photonadded coherent states of light. science, 306(5696):660-662, 2004.

[52] Nguyen Ba An. Multimode higher-order antibunching and squeezing in trio coherent states. Journal of Optics B: Quantum and Semiclassical Optics, 4(3):222, 2002.

[53] A Pathak and ME Garcia. Control of higher order antibunching. Applied Physics B, 84(3):479-484, 2006.

[54] Mark Hillery. Amplitude-squared squeezing of the electromagnetic field. Physical Review A, 36(8):3796, 1987.

[55] GS Agarwal and K Tara. Nonclassical character of states exhibiting no squeezing or sub-poissonian statistics. Physical Review A, 46(1):485, 1992. 
[56] Evgeny V Shchukin and Werner Vogel. Nonclassical moments and their measurement. Physical Review A, 72(4):043808, 2005.

[57] Héctor Moya-Cessa and Peter L Knight. Series representation of quantum-field quasiprobabilities. Physical Review A, 48(3):2479, 1993.

[58] Anatole Kenfack and Karol Życzkowski. Negativity of the wigner function as an indicator of non-classicality. Journal of Optics B: Quantum and Semiclassical Optics, 6(10):396, 2004.

[59] K Banaszek, C Radzewicz, K Wódkiewicz, and JS Krasiński. Direct measurement of the wigner function by photon counting. Physical Review A, 60(1):674, 1999.

[60] Patrice Bertet, Alexia Auffeves, Paolo Maioli, Stefano Osnaghi, Tristan Meunier, Michel Brune, Jean-Michel Raimond, and Serge Haroche. Direct measurement of the wigner function of a one-photon fock state in a cavity. Physical Review Letters, 89(20):200402, 2002.

[61] Yoni Shalibo, Roy Resh, Ofer Fogel, David Shwa, Radoslaw Bialczak, John M Martinis, and Nadav Katz. Direct wigner tomography of a superconducting anharmonic oscillator. Physical Review Letters, 110(10):100404, 2013.

[62] DT Smithey, M Beck, Michael G Raymer, and A Faridani. Measurement of the wigner distribution and the density matrix of a light mode using optical homodyne tomography: Application to squeezed states and the vacuum. Physical Review Letters, 70(9):1244, 1993.

[63] Sergey N Filippov and Vladimir I Man'ko. Optical tomography of fock state superpositions. Physica Scripta, 83(5):058101, 2011.

[64] I.S. Gradshteyn and I.M. Ryzhik. Table of Integrals, Series, and Products. Academic Press, 2001. 


\section{APPENDIX: A}

\section{Detailed derivation of Wigner function}

Fock state $|n\rangle$ can be written in position space as

$$
|n\rangle=\phi_{n}(x)=b_{n} \mathrm{e}^{-\frac{x^{2}}{2}} H_{n}(x),
$$

where $b_{n}=\frac{1}{\pi^{\frac{1}{4}}\left[2^{n} n !\right]^{\frac{1}{2}}}, \hbar=1$ and $H_{n}(x)$ is the Hermite polynomial. Consequently, we can express the FSS 17 as

$$
\psi(x)=\sum_{n=0}^{N} c_{n} \phi_{n}(x) .
$$

Therefore, the Wigner function of an arbitrary FSS in the integral form is

$$
\begin{aligned}
W\left(x, p^{\prime}\right) & =\frac{1}{\pi} \int_{-\infty}^{\infty} \psi^{*}(x+y) \psi(x-y) \exp \left(2 i p^{\prime} y\right) d y \\
& =\frac{1}{\pi} \sum_{n, n^{\prime}=0}^{N} c_{n}^{*} c_{n^{\prime}} \int_{-\infty}^{\infty} \phi_{n}^{*}(x+y) \phi_{n^{\prime}}(x-y) \exp \left(2 i p^{\prime} y\right) d y \\
& =\frac{1}{\pi} \sum_{n, n^{\prime}=0}^{N} c_{n}^{*} c_{n^{\prime}} W_{n n^{\prime}}\left(x, p^{\prime}\right),
\end{aligned}
$$

where

$$
W_{n n^{\prime}}\left(x, p^{\prime}\right)=\int_{-\infty}^{\infty} \phi_{n}^{*}(x+y) \phi_{n^{\prime}}(x-y) \exp \left(2 i p^{\prime} y\right) d y .
$$

Using (A-1), we can write

$$
\begin{aligned}
W_{n n^{\prime}}\left(x, p^{\prime}\right) & =b_{n}^{*} b_{n^{\prime}} \int_{-\infty}^{\infty} \mathrm{e}^{-\frac{(x+y)^{2}}{2}} H_{n}(x+y) \mathrm{e}^{-\frac{(x-y)^{2}}{2}} H_{n^{\prime}}(x-y) \mathrm{e}^{2 i p^{\prime} y} d y \\
& =b_{n}^{*} b_{n^{\prime}} \int_{-\infty}^{\infty} H_{n}(x+y) H_{n^{\prime}}(x-y) \mathrm{e}^{-x^{2}-y^{2}+2 i p^{\prime} y} d y \\
& =b_{n}^{*} b_{n^{\prime}} \mathrm{e}^{-x^{2}} \int_{-\infty}^{\infty} H_{n}(x+y) H_{n^{\prime}}(x-y) \mathrm{e}^{-\left(y-i p^{\prime}\right)^{2}-p^{\prime 2}} d y \\
& =b_{n}^{*} b_{n^{\prime}} \mathrm{e}^{-\left(x^{2}+p^{\prime 2}\right)} I_{n n^{\prime}}
\end{aligned}
$$

where

$$
I_{n n^{\prime}}=\int_{-\infty}^{\infty} H_{n}(x+y) H_{n^{\prime}}(x-y) \mathrm{e}^{-\left(y-i p^{\prime}\right)^{2}} d y .
$$

Writing $y-i p^{\prime}=y^{\prime}$ in $\mathrm{A}-5$ we obtain

$$
\begin{aligned}
I_{n n^{\prime}} & =\int_{-\infty}^{\infty} H_{n}\left(x+y^{\prime}+i p^{\prime}\right) H_{n^{\prime}}\left(x-y^{\prime}-i p^{\prime}\right) \mathrm{e}^{-\left(y^{\prime}\right)^{2}} d y^{\prime} \\
& =(-1)^{n^{\prime}} \int_{-\infty}^{\infty} H_{n}\left(y^{\prime}+\left(x+i p^{\prime}\right)\right) H_{n^{\prime}}\left(y^{\prime}+\left(i p^{\prime}-x\right)\right) \mathrm{e}^{-\left(y^{\prime}\right)^{2}} d y^{\prime} \\
& =(-1)^{n^{\prime}} 2^{n^{\prime}} \pi \frac{1}{2} n !\left(i p^{\prime}-x\right)^{n^{\prime}-n} L_{n}^{n^{\prime}-n}\left(-2 y\left(i p^{\prime}-x\right)\right), \quad n \leq n^{\prime} .
\end{aligned}
$$

In the last step, we have used the identity 7.377 of 64 . Now substituting $(A-4)$ and $(A-6)$ in $A-2$ we obtain

$$
W\left(x, p^{\prime}\right)=\frac{1}{\pi^{\frac{1}{2}}} \sum_{n, n^{\prime}=0}^{N} c_{n}^{*} c_{n^{\prime}} b_{n}^{*} b_{n^{\prime}} \mathrm{e}^{-\left(x^{2}+p^{\prime 2}\right)}(-1)^{n^{\prime}} 2^{n^{\prime}} n !\left(i p^{\prime}-x\right)^{n^{\prime}-n} L_{n}^{n^{\prime}-n}\left(-2 y\left(i p^{\prime}-x\right)\right), \quad n \leq n^{\prime} .
$$

